\title{
High Temporal and Spatial Resolution Distributed Fiber Bragg Grating Sensors Using Time-Stretch Frequency-Domain Reflectometry
}

\author{
Eamonn J Ahmad, Chao Wang, Member, IEEE, Dejun Feng, Zhijun Yan, and Lin Zhang
}

\begin{abstract}
A novel interrogation technique for fully distributed linearly chirped fiber Bragg grating (LCFBG) strain sensors with simultaneous high temporal and spatial resolution based on optical time-stretch frequency-domain reflectometry (OTS-FDR) is proposed and experimentally demonstrated. LCFBGs is a promising candidate for fully distributed sensors thanks to its longer grating length and broader reflection bandwidth compared to normal uniform FBGs. In the proposed system, two identical LCFBGs are employed in a Michelson interferometer setup with one grating serving as the reference grating whereas the other serving as the sensing element. Broadband spectral interferogram is formed and the strain information is encoded into the wavelength-dependent free spectral range (FSR). Ultrafast interrogation is achieved based on dispersion-induced time stretch such that the target spectral interferogram is mapped to a temporal interference waveform that can be captured in real-time using a single-pixel photodector. The distributed strain along the sensing grating can be reconstructed from the instantaneous RF frequency of the captured waveform. High-spatial resolution is also obtained due to high-speed data acquisition. In a proof-of-concept experiment, ultrafast real-time interrogation of fully-distributed grating sensors with various strain distributions is experimentally demonstrated. An ultrarapid measurement speed of $50 \mathrm{MHz}$ with a high spatial resolution of $31.5 \mu \mathrm{m}$ over a gauge length of $25 \mathrm{~mm}$ and a strain resolution of $9.1 \mu \varepsilon$ have been achieved.
\end{abstract}

Index Terms - Chromatic dispersion, fiber Bragg grating, Fourier transforms, optical interferometry, frequency-domain reflectometry, time stretch.

Manuscript received 16 May 2016; revised 11 August 2016; accepted 15 August 2016. This work was supported in part by EU FP7 Marie-Curie Career Integration Grant under Grant 631883, and in part by the Royal Society Research Grants under Grant RG150036.

E. J. Ahmad and C. Wang are with the School of Engineering and Digital Arts, University of Kent, Canterbury CT2 7NT, U.K. (e-mail: eja6@kent.ac.uk; c.wang@kent.ac.uk).

D. Feng is with the School of Information Science and Engineering, Shandong University, Jinan 250100, China, and also with the School of Engineering and Digital Arts, University of Kent, Canterbury CT2 7NT, U.K. (e-mail: dejunfeng@sdu.edu.cn).

Z. Yan was with the Aston Institute of Photonic Technologies, Aston University, Birmingham B4 7PG, U.K., and is currently with the State Key Laboratory of Transient Optics and Photonics, Xi'an Institute of Optics and Precision Mechanics, Chinese Academy of Sciences, Xi'an 710119, China (e-mail: yanzhijun@gmail.com).

L. Zhang is with the Aston Institute of Photonic Technologies, Aston University, Birmingham B4 7PG, U.K. (e-mail: l.zhang@aston.ac.uk).

\section{INTRODUCTION}

$\mathrm{P}$ RECISE measurement of strain, temperature, refractive index, and other chemical and biomedical parameters using sensors is an essential requirement in various applications pertaining to civil engineering, structural health monitoring and biomedical screening. Optical fiber Bragg gating (FBG) sensors offer significant advantages over conventional electrical sensors, such as small size, light weight, immunity to electromagnetic interference, low cost, high durability and multiplexing capability [1], [2]. The sensing information is usually directly encoded as the grating wavelength change. Interrogation of FBG sensors, which monitors the Bragg wavelength shift and translates the wavelength shift to the applied strain or temperature change, is an essential part of the whole optical sensor systems. While great progress has been made in interrogation of FBG sensors in the past few decades with various developed techniques [3-7], most efforts have overwhelmingly placed emphasis on improving the wavelength resolution, leading to impressive ultra-sensitive interrogation system with sub-pm resolution [8].

Mostly overlooked in FBG sensors is the temporal resolution of interrogation systems, which is however of paramount importance in a diverse range of scientific and engineering applications. For instance, an ultrafast FBG interrogation system is an indispensable tool for studying dynamic phenomena such as chemical dynamics and neural activity, and monitoring fast vibrating objects such as aircraft engine diagnostics. High-speed interrogation of FBG sensors has become a research topic of great interest recently [9]. The use of Fourier domain mode-locked swept laser [10], [11] has enabled interrogation speed up to $100 \mathrm{kHz}$. Most recently, a real-time spectroscopy technique [12], [13] has been successfully used in FBG sensor interrogation leading to impressive ultrafast and single-shot measurement at speed of tens of $\mathrm{MHz}$ [14-18]. This is made possible by using chromatic dispersion to map the instantaneous spectrum of an ultrashort optical pulse to a temporal waveform based on wavelength-to-time mapping or dispersive Fourier transformation [19], [20], which can be captured in real-time using a single high-speed photodetector. This technique has been successfully applied for sensing of strain and pressure in dynamic extremes of materials [21]. In addition, research efforts have been made to improve the interrogation resolution and signal-to-noise ratio [22], and to 
overcome the fundamental tradeoff between the interrogation speed and resolution [23] in real-time spectroscopy based interrogation systems.

However, the previously reported high-speed FBG sensor interrogation systems [14-18], [21-23] all fall short in interrogation of fully-distributed FBG sensors: they can only measure the average value of strain or temperature change over the length of the FBG sensor. While quasi-distributed strain sensing can be implemented by multiplexing many discrete FBG sensors, high spatial resolution fully-distributed measurement of strain and temperature over a short gauge length within an FBG is required in structure health monitoring, such as crack detection. A linearly chirped fiber Bragg grating (LCFBG) is a promising candidate for fully distributed sensors thanks to its longer grating length and broader reflection bandwidth compared to normal uniform FBGs. The local Bragg wavelength of the LCFBG directly relates with the distributed sensing information along the grating, enabling fully-distributed intra-grating sensing based on wavelength demodulation. Another advantage of using LCFBGs compared to long-length uniform FBGs is that the LCFBG also serves as a dispersive element in the photonic time-stretch scheme, which reduces the requirement for lossy dispersive fibers and hence enhances the signal-to-noise ratio. Several interrogation techniques for fully-distributed LCFBG sensors have been proposed and demonstrated. Direct spectrum measurement [24] is simple and straightforward as the local strain changes cause spectral dips, but the spatial resolution is poor and spectrum measurement is slow. A fiber loop ring-down (FLRD) spectroscopy has been proposed to interrogate LCFBG sensors with $2 \mathrm{~mm}$ spatial resolution and $1 \mathrm{kHz}$ temporal resolution [25]. A higher spatial resolution of $300 \mu \mathrm{m}$ has been achieved using time domain low-coherence reflectometry (LCR) [26]. However, the measurement speed was limited by mechanical movement of the reference beam and signal-to-noise ratio is poor due to the use of low-coherence optical source. Distributed strain measurement with spatial resolution of $4 \mathrm{~mm}$ has been demonstrated using LCFBGs and optical frequency-domain reflectometry (OFDR) [27]. No mechanical movement is required, but the interrogation speed is limited by the laser wavelength sweeping and falls below $\mathrm{kHz}$ range.

In this paper, we propose a novel interrogation technique for fully-distributed LCFBG sensors that achieves simultaneous high temporal and spatial resolution based on optical time-stretch frequency-domain reflectometry (OTS-FDR). In the proposed system, two identical LCFBGs are employed in a Michelson interferometer setup with one grating serving as the reference grating whereas the other serving as the sensing element. Broadband spectral interferogram is formed and the distributed strain information that is related with the local Bragg wavelength is now encoded into the local wavelength dependent free spectral range (FSR). In the proposed OTS-FDR system, the target spectral interferogram is mapped to a temporal interference waveform that can be captured in real-time using a high-speed single-pixel photodector. Therefore ultrafast and single-shot interrogation of LCFBG sensors is achieved with the interrogation speed identical to the repetition rate of the pulsed laser. The distributed strain information along the sensing grating is reconstructed from the instantaneous RF frequency of the captured waveform by applying short-time Fourier transform (STFT) analysis. High-spatial resolution is also obtained thanks to high-speed data acquisition. Note that although a similar interrogation setup has been used in a previous system [23], only average strain change was measured by using normal Fourier transform. Here we achieve fully-distributed strain measurement by applying time/space resolved STFT analysis in the OTS-FDR scheme for the first time to the best of our knowledge. Ultrafast real-time interrogation of fully-distributed grating sensors with various strain distributions at measurement speed of $50 \mathrm{MHz}$ with a high spatial resolution of $31.5 \mu \mathrm{m}$ over a gauge length of $25 \mathrm{~mm}$ and a high strain resolution of $9.1 \mu \varepsilon$ is experimentally demonstrated.

The remainder of this paper is structured as follows. In Section II, we first describe the operation principle of the proposed distributed LCFBG sensors interrogation system. The relationship between the instantaneous RF frequency change of the interference pattern and the local strain within the LCFBG sensor is established. A proof-of-concept experimental demonstration is performed in Section III. Ultrafast and high spatial resolution interrogation of LCFBG strain sensors with both uniform and non-uniform strain change is achieved. In Section IV, discussions on system performance in terms of temporal and spatial resolution and strain measurement sensitivity are provided. Finally, we summarize and conclude our work in Section V.

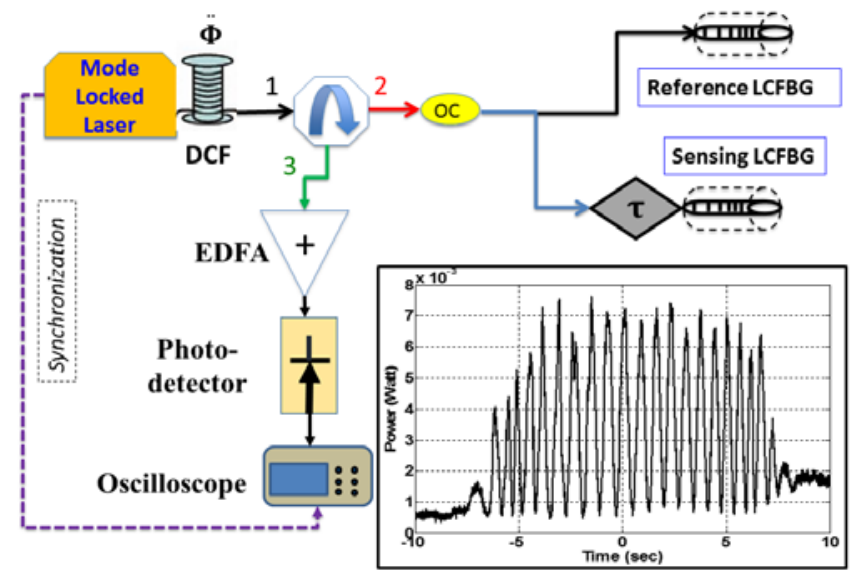

Fig. 1. The proposed ultrafast and high spatial resolution LCFBG interrogation system based on optical time stretch frequency domain reflectormetry (OTS-FDR). Inset: the measured initial temporal interference waveform with no strain applied. EDFA: erbium-doped fiber amplifier, DCF: dispersion compensating fiber, OC: optical coupler, LCFBG: linealry chirped fiber Bragg grating.

\section{PRINCIPLE}

An FBG is a single mode optical fiber that can be constructed 
by periodically varying the refractive index profile along a certain length of the fiber via UV illumination [1]. The design of an FBG allows a specific wavelength of light, called the Bragg wavelength, to be reflected while transmitting all others. The Bragg wavelength can be calculated as follows:

$$
\lambda_{B}=2 n_{\text {eff }} \Lambda
$$

where $\Lambda$ is the grating period and $n_{\text {eff }}$ is the effective refractive index. A change in strain and or temperature will change both the refractive index and the grating period. Therefore most FBG sensors are wavelength modulated.

LCFBGs have a linearly varying grating period along its length. They exhibit a broadband reflection spectrum and each Bragg wavelength in the reflected spectrum is related with distinct positions along the grating length. Therefore, LCFBG is a promising candidate for fully-distributed sensors over short gauge length within the grating. Without loss of generality, only strain change is considered in the following analysis. However the proposed interrogation method also works for temperature sensing. A change in local strain $\varepsilon(z)$ modifies the specific Bragg wavelength at the local position $z$,

$$
\Delta \lambda_{B}(z)=\lambda_{B}(z)\left(1-\rho_{e}\right) \varepsilon(z)
$$

where $\rho_{\mathrm{e}}$ is the strain-optic coefficient of the optical fiber.

In order to interrogate the local Bragg wavelength change $\Delta \lambda_{B}(z)$, an ultrafast and high spatial resolution interrogation approach based on OTS-FDR is proposed, as shown in Fig. 1. A Michelson interferometer setup is constructed using two identical LCFBGs with one serving as the sensing grating subjected to applied distributed strain, and the other as the reference grating free from any strain. Optical interference is formed and a broadband spectral interferogram is obtained with its free spectral range (FSR) determined by the initial time delay $T_{0}$ between the two arms, which can be controlled using a variable optical delay line (VODL) in one arm of the optical interferometer. The inset in Fig. 1 shows a measured initial temporal interference waveform with no strain applied.

A passively mode-locked laser (MLL) is used to generate highly-coherent ultrashort optical pulses with a broad optical spectrum. The optical pulse is first stretched by a dispersion compensating fiber (DCF) and then sent to the Michelson interferometer. An optical time-stretch frequency domain reflectometry is thus formed and the spectral interferogram is mapped to a temporal interference waveform thanks to dispersion-induced wavelength-to-time mapping, also known as dispersive Fourier transform. In the OTS-FDR scheme, the central frequency of the mapped temporal waveform is determined by the initial time delay and the overall chromatic dispersion $\ddot{\Phi}\left(\right.$ in $\mathrm{ps}^{2}$ ) of the DCF, which is given by [23]

$$
f_{R F}=\frac{T_{0}}{\ddot{\Phi}}
$$

When a local strain $\varepsilon(z)$ is applied to the sensing LCFBG with (a)

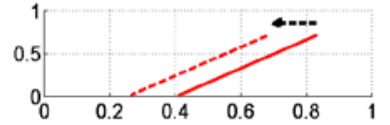

(c)

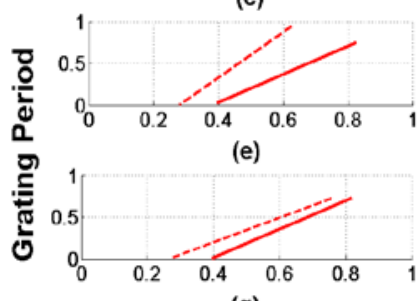

(g)

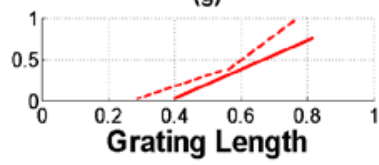

(b)

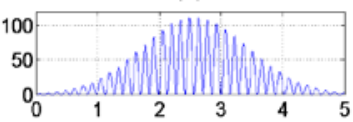

(d)

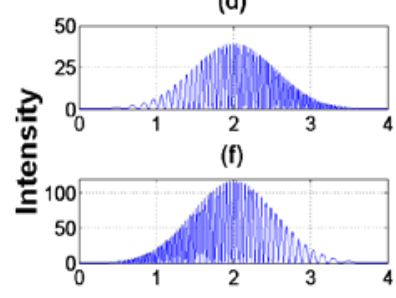

(h)

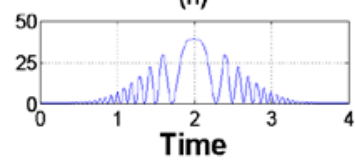

Fig. 2. Comparison between redistribution of local Bragg wavelength in the sensing grating (dashed lines) with respect to the reference grating (solide lines) for (a) uniform, and (c), (e) and (g) non-uniform strainsapplied. Figures (b), (d), (f) and (h) show the resulting temporal waveforms.

a chirp rate $C(\mathrm{~nm} / \mathrm{cm})$, the change of local Bragg wavelength $\Delta \lambda_{B}(z)$ introduces a time delay change, $\Delta \tau$, given by [28]

$$
\Delta \tau=\frac{2 n_{\text {eff }}}{c \times C} \Delta \lambda_{B}(z)
$$

where $c$ is the speed of light in air, and $n_{\text {eff }}$ is the refractive index of fiber core. This extra temporal shift $\Delta \tau$ is added to the overall time delay between two interferometer arms and leads to the change of instantaneous RF frequency, $\Delta f_{R F}(t)$, in the temporal interference waveform. According to (2)-(4), we have

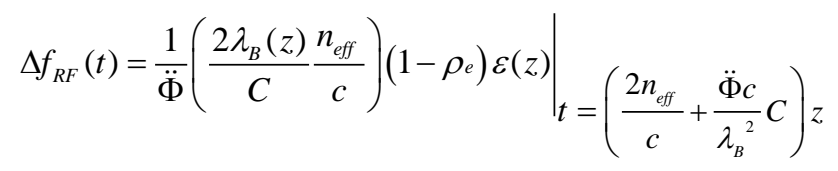

Therefore, as shown in Eq. (5), fully distributed or local strain information along the LCFBG sensor can be demodulated from the change of instantaneous RF frequency thanks to the unique and linear one-to-one mapping relation between spatial position, Bragg wavelength and time. Ultrafast interrogation speed is identical to the repetition rate of the pulsed laser. The spatial resolution is determined by the temporal resolution of instantaneous RF frequency measurement. To summarize, the strain value is measured from the change of instantaneous RF frequency and location is measured from the instant time. Ultrafast single-shot measurement is enabled due to the pulsed nature of broadband coherent optical source.

The proposed interrogation approach can be explained in an intuitive way by looking at the change of grating chirp due to the applied strain. Fig. 2 (a) depicts the effect of uniform strain applied to the sensing LCFBG, resulting in a redistribution of the period while maintaining the same chirp rate. Beating the two time delayed and stretched optical pulses at a PD yields a temporal waveform with a constant RF frequency across the 
pulse duration, as shown in Fig. 2(b). The change of RF frequency compared to the strain-free case can be used to detect the uniform strain applied. When nonuniform strain gradient is applied to the sensing LCFBG, its chirp rate will be changed differently with respected to the reference grating according to the local strain, resulting in chirped waveforms with a monotonic increase or decrease in instantaneous RF frequency [29], as shown in Figs. 2 (c-f). On the other hand, a nonlinear strain gradient, wherein a part of the grating is non-uniformly stretched and the other part is non-uniformly compressed, produces a beating waveform with its frequency chirp rate being swapped, as can be observed in Figs. 2(g) and (h).

\section{EXPERIMENT}

To verify the utility of the proposed technique, a proof-of-concept experiment based on the setup shown in Fig. 1 is performed. A passively mode-locked fiber laser (Calmar MENDOCINO FPL) is employed as the optical source to generate a transform-limited Gaussian pulse train with a repetition rate of $50 \mathrm{MHz}$. The ultrashort optical pulse has a full-width at half-maximum (FWHM) pulse width of $800 \mathrm{fs}$, a 3-dB spectral bandwidth of $16 \mathrm{~nm}$ and a central wavelength of $1550 \mathrm{~nm}$. Two $2.5 \mathrm{~cm}$ long LCFBGs with an identical center wavelength of $1554.5 \mathrm{~nm}$ and 3-dB bandwidth of $7 \mathrm{~nm}$ are employed as the sensing and reference gratings. A VODL is inserted in the sensing arm of the interferometer to control the initial time delay difference between two arms. A DCF with total group velocity dispersion (GVD) of $\ddot{\Phi}=11520 \mathrm{ps}^{2}$ is used for optical time-stretch process. The mapped temporal interference waveform is detected by a $53 \mathrm{GHz}$ photodetector and recorded using a high-speed sampling oscilloscope (Agilent 86100A).
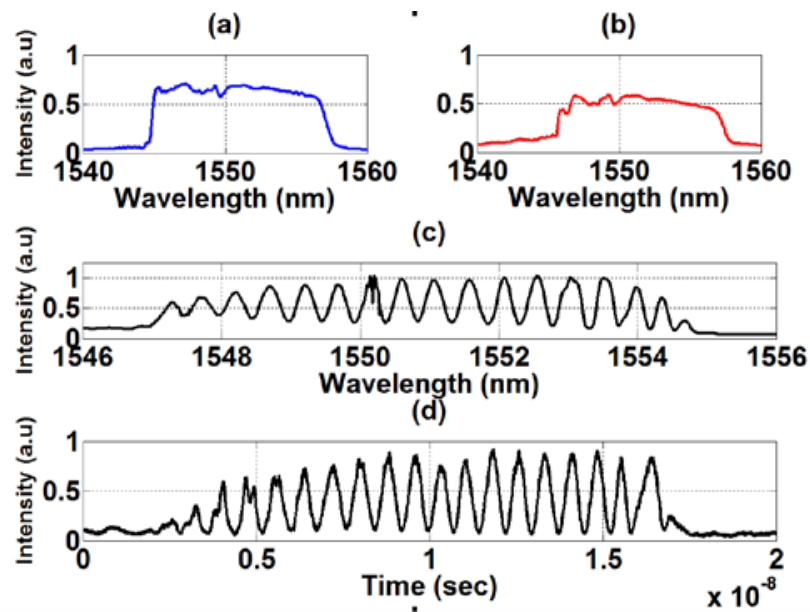

Fig. 3. Measured basic OTS-FDR performance with no strain applied. (a) Reflection spectrum from the reference LCFBG, (b) reflection spectrum from the sensing grating, (c) spectral interferogram, (d) temporal inteference waveform verifying the dispersion-induced wavelength-to-time mapping.

Fig. 3 shows the measured basic OTS-FDR performance when no strain is applied. Figs. 3(a) and (b) depict the reflected spectra of the reference and sensing LCFBGs, respectively. A spectral interferogram is formed from overlapping the reflected spectra from the sensing and reference LCFBGs, as shown in Fig. 3 (c). The measured temporal waveform after optical time stretch is shown in Fig. 3(d), which essentially implies that under the temporal far-field Fraunhofer condition, the dispersion-induced wavelength-to-time mapping is perfectly achieved. Note that as the DCF provides negative GVD, longer wavelength will have shorter time delay (traveling faster).

\section{A. Uniform Strain measurement for system characterization}

The inset in Fig. 1 shows the measured initial temporal interference waveform when no strain is applied. To characterize the interrogation system's response, the sensing LCFBG was first uniformly stretched with different applied strains, which generates single-tone temporal waveforms with different uniform RF frequencies. Fig. 4 shows the measured RF frequency as a function of the applied uniform strains. A good linear relation is obtained, as predicted by Eq. (5). Spectrograms of two recorded waveforms are calculated using STFT analysis and shown in Fig. 4 for uniform strain values of 180 and $625 \mu \varepsilon$, respectively. It is also verified that the instantaneous RF frequency is almost constant across the whole waveform. From linear fitting of the measured data, a frequency change to applied strain ratio of $5.5 \mathrm{MHz} / \mu \varepsilon$ is obtained, which matches perfectly with the theoretical value of $5 \mathrm{MHz} / \mu \varepsilon$ as predicted by Eq. (5) and proves the high sensitivity of this technique in decoding variations in strain. Fig. 4 also serves as a system characterization, through which the strain value can be unambiguously determined as long as the change of instantaneous RF frequency is measured.

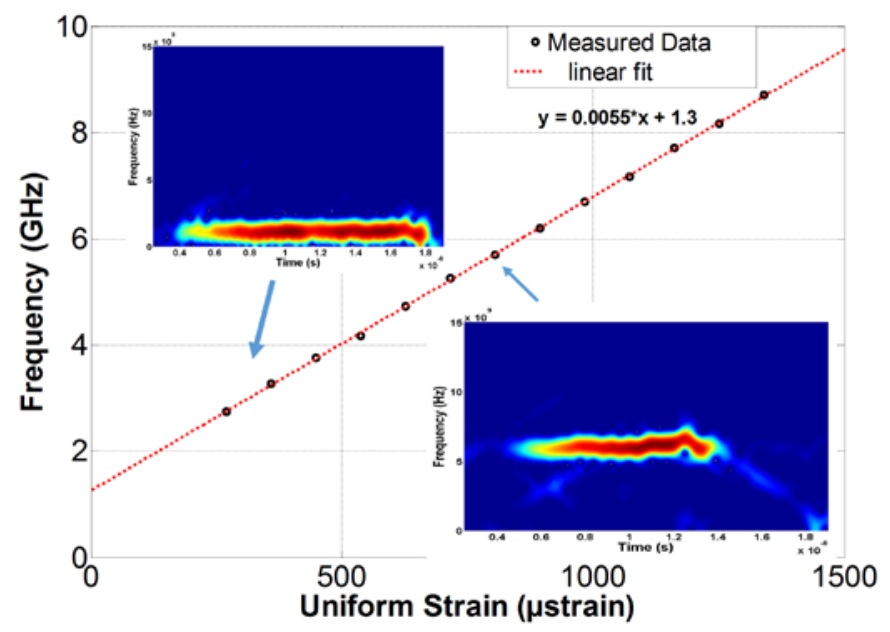

Fig. 4. Characterization of the interrogation system by applying various uniform strains. Insets show spectrograms of the temporal interference patterns at uniform strain values of 180 and $625 \mu \varepsilon$, respectively. A linear fitting result is also shown in red solid line.

\section{B. Nonuniform expansion and compression}

In order to demonstrate the capability of our interrogation technique to monitor fully-distributed strain, more experiments have been done to apply nonuniform expansion and 
compression along the sensing LCFBG with a uniform strain gradient. We glue the grating onto an elastic and flexible substrate. Initially the substrate is bent to a parabolic shape. Therefore the sensing LCFBG on the top surface of the substrate will suffer from nonuniform expansion. As the longer wavelength part of the grating is closer to the centre of the parabolic shape substrate, it will be stretched more than the shorter wavelength part. The grating period within the sensing LCFBG is remodified as depicted in Fig. 2 (e). Fig. 5(a) shows the generated temporal interference waveform with a negative frequency chirp, as verified by its spectrogram shown in Fig. 5(b). In a second case, the substrate is bent to an inverse parabolic shape. Due to the applied nonuniform compression along the sensing LCFBG with larger wavelength part under more compression, a positively chirped temporal waveform is obtained as shown in Fig. 5(c) and its spectrogram depicting instantaneous RF frequency is shown in Fig. 5(d). The change in instantaneous RF frequency at a particular instant of time reflects the distributed nonuniform strain and can be mapped to the spatial location along the length of the LCFBG according to Eq. (5).

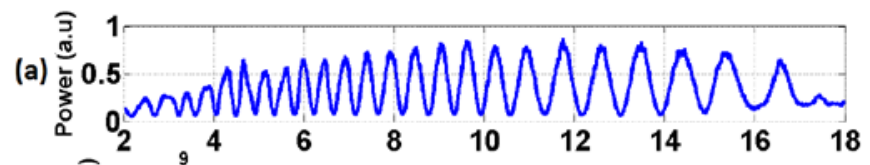

(b)

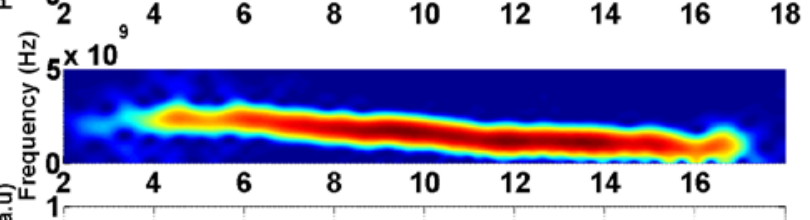

(c)

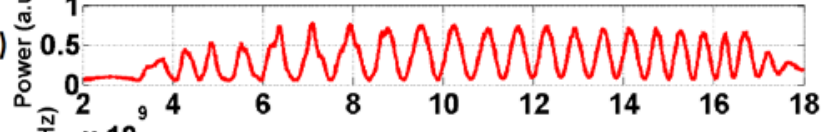

(d)

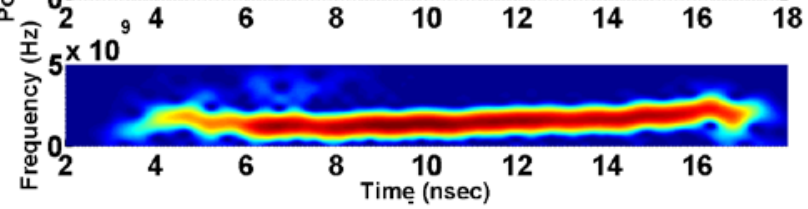

Fig. 5. Demonstration of nonuniform strain sensing. (a, c) The measured temporal waveforms, and (b, d) their spectrograms for nonuniform expansion and compressoin of the sensing LCFBG, respectively.

\section{S-shape bending with changing strain gradient}

In order to further demonstrate the effect of local stress with changing strain gradient along the sensor grating, the substrate is bent to an S-shape, enabling part of the grating period to be nonuniformly stretched and the other part expanded. Such a strain profile results in a change in the grating chirp rate of the sensing LCFBG, as depicted in Fig. 2 (g). This differential local expansion and contraction of the grating period will be translated to the different slopes of frequency sweep in the beating temporal waveform, as illustrated in Fig. 2(h).

An experiment has been carried out to verify this concept. Fig. 6 depicts the measured temporal beating waveform and its corresponding spectrogram under the applied nonuniform strain gradient. Longer Bragg wavelength part of the grating is under nonuniform expansion, leading to negative sweep rate of (a)
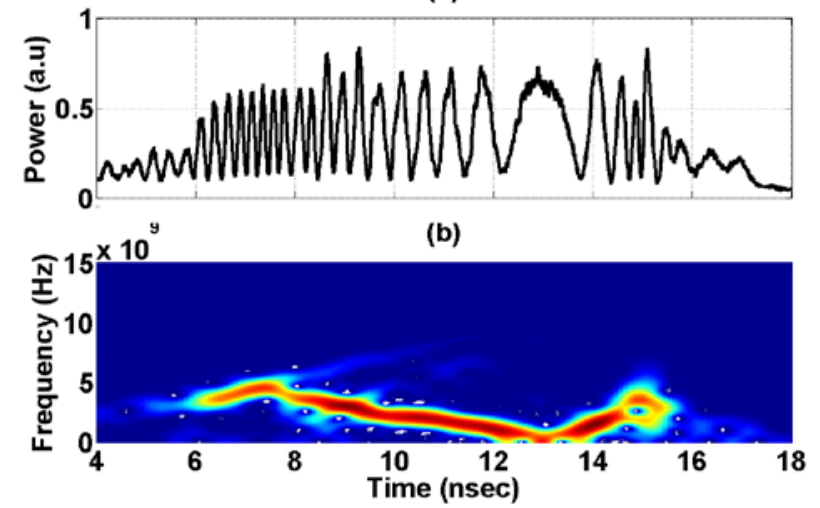

Fig. 6. Demonstration of changing strain gradient by applying S-shape bending. (a) The measured temporal intereference waveform and (b) its spectrogram showing different frequency sweeping rates.

instantaneous RF frequency during the first (left) part of waveform. A change in the frequency chirp rates denotes a turning point, from which the remaining part of grating is under nonuniform compression. The instant time of change in chirp rates is related to the spatial location of the turning point following the time-to-location mapping in Eq. (5).

(a)

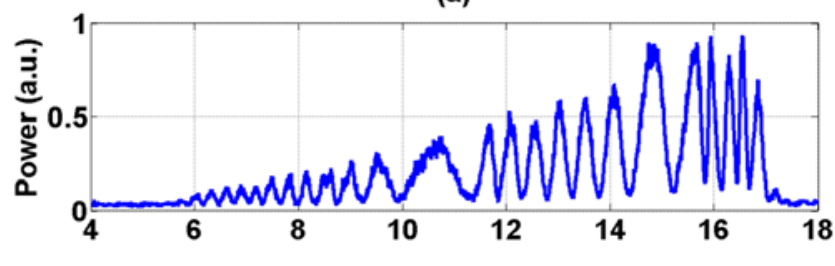

(b)

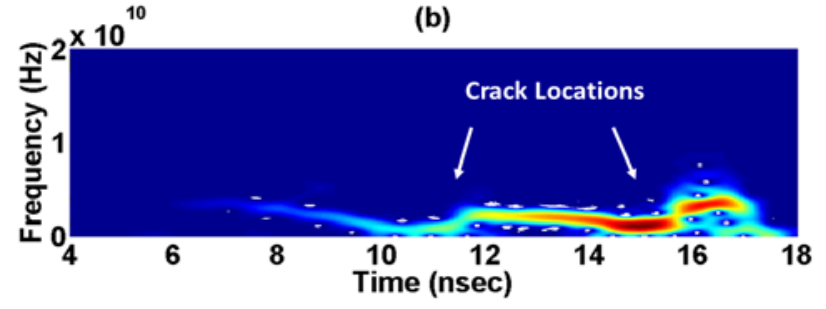

Fig. 7. Demonstration of local crack detection. (a) The measured temporal intereference waveform and (b) its spectrogram clearly showing two frequency hoppings which identifies the locations of the two holes.

\section{Crack detection}

The high strain sensitivity and high spatial resolution of the proposed interrogation technique provide sufficient motivation to explore the capability of LCFBGs to identify cracks or dislocations along the substrate onto which the sensor grating is mounted. In order to demonstrate this aspect, we drilled two holes with diameter of $2 \mathrm{~mm}$ separated by $10 \mathrm{~mm}$ along the centre axis of the substrate and glued the sensing LCFBG on top of the substrate crossing the holes. A nonuniform expansion of the whole sensing grating was performed. The two short sections of the LCFBG that fell into the hole areas acted as dividing points and two sides of each point are subjected to different strain change. Based on the theory of OTS-FDR as explained above, a sudden instantaneous frequency change is expected at the specific instant time corresponding to each 
individual holes. Fig. 7(a) shows the recorded temporal beating waveform and a measure of the instantaneous RF frequency along time can be observed from Fig. 7 (b). The two jumps in instantaneous frequency is a clear evidence that the two holes have been detected and their locations are determined from the instant time of the frequency hopping according to Eq. (5).

\section{DISCUSSIONS}

In our proposed interrogation system, the local strain information can be interrogated from the change of instantaneous RF frequency of the beating temporal waveform. Eliminating the slow spectrum measurement [25-27], OTS-FDR based technique features much faster interrogation speed, which is identical to the repetition rate of the pulsed laser. In this work, high temporal resolution of $50 \mathrm{MHz}$ is obtained, which is several orders of magnitude higher than conventional OFDR methods.

On the other hand, high spatial resolution of the proposed interrogation method is assured due to the fact that the temporal interference waveform can be captured using a high-speed PD and a fast analog-to-digital converter. Therefore, the instantaneous RF frequency can be determined with very high temporal resolution. In this work, an equivalent temporal sampling rate of $67.5 \mathrm{GS} / \mathrm{s}$ was used to detect the interference waveforms, leading to a maximum temporal resolution of 14.8 ps in theory. According to the linear time-wavelength-location mapping as shown in Eq. (5), the spatial resolution is estimated as high as $31.5 \mu \mathrm{m}$ in principle.

In FBG sensors, the strain response or sensitivity is approximately $1.15 \mathrm{pm} / \mu \varepsilon$ at the wavelength band of $1550 \mathrm{~nm}$. So measuring very weak strain is a real challenge in conventional FBG interrogation systems due to the limited spectral resolution of optical spectrum measurement instrument. In the proposed interrogation system, the strain sensitivity is measured as $5 \mathrm{MHz} / \mu \varepsilon$. The strain information is encoded to the change of RF frequency, in contrast to optical wavelength in conventional systems, and can be measured with much higher accuracy using a RF spectrum analyzer. The frequency resolution of our system, which is defined by the 3-dB bandwidth of RF spectrum for a single-tone interference waveform, is essentially limited by the repetition rate of the pulsed laser [29], which is $50 \mathrm{MHz}$ in this work. Therefore, the strain resolution of the proposed system is estimated to be 9.1 $\mu \varepsilon$.

Conventional FBG sensors cannot distinguish wavelength shifts caused by strain and temperature. One solution for simultaneous measurement of both strain and temperature in photonic time stretch scheme is to use a high-birefringence LCFBG [30]. In the proposed system, two LCFBGs are used, which not only form a Michelson interferometer for FDR detection, but also eliminate the cross-sensitivity to temperature. Since two gratings are placed in close proximity, temperature change will lead to an identical wavelength shift, making the beat RF frequency only dependent on the applied strain.

Finally, it is also worth noting that as STFT analysis is used to estimate the instantaneous RF frequency over time, the maximum temporal resolution (and hence the spatial resolution) is achieved with a sacrificed frequency resolution, which in turn leads to a poor accuracy in strain measurement. A fundamental trade-off between frequency and temporal resolution in STFT analysis always exists.

\section{CONCLUSION}

We have proposed and experimentally demonstrated a novel interrogation technique for fully-distributed FBG strain sensors with simultaneous high temporal and spatial resolution. This is made possible by applying dispersion-induced optical time stretch frequency domain reflectometry (OTS-FDR). The distributed strain information along the grating length can be reconstructed from the recorded temporal interference waveform. The strain value was measured from the change of instantaneous RF frequency and location was measured from the instant time. The proposed technique is verified by proof-of-concept experiments, in which measurements of uniform strain, nonuniform expansion and compression, changing strain gradient and local crack detection have been demonstrated. An ultrarapid temporal resolution of $50 \mathrm{MHz}$, a high spatial resolution of $31.5 \mu \mathrm{m}$ over a gauge length of $25 \mathrm{~mm}$ and a strain resolution of $9.1 \mu \varepsilon$ have been achieved. Thanks to its simultaneous ultrafast speed, high spatial resolution, and sensitive strain response, we believe the proposed technique is a promising interrogation solution for short range fully-distributed sensing systems where ultrafast and ultrahigh spatial resolution measurement is required.

\section{REFERENCES}

[1] A. D. Kersey, M. A. Davis, H. J. Patrick, M. LeBlanc, K. P. Koo, C. G. Askins, M. A. Putnam, and E. J. Friebele, "Fiber grating sensors," $J$. Lightwave Technol., vol. 15, no. 8, pp. 1442-1463, 1997.

[2] Y. J. Rao, "In-fiber Bragg grating sensor," Meas. Sci. Technol., vol. 8, no. 4, pp. 355-375, 1997.

[3] S. H. Yun, D. J. Richardson, and B. Y. Kim, "Interrogation of fiber grating sensor arrays with a wavelength-swept fiber laser," Opt. Lett. vol. 23, no. 11, pp. 843-845, 1998.

[4] A. D. Kersey, T. A. Berkoff, and W. W. Morey, "Multiplexed fiber Bragg grating strain-sensor system with a fiber Fabry-Pérot wavelength filter," Opt. Lett., vol. 18, no. 16, pp. 1370-1372, 1993.

[5] M. Song, S. Yin, and P. B. Ruffin, "Fiber Bragg grating strain sensor demodulation with quadrature sampling of a Mach-Zehnder interferometer,” Appl. Opt., vol. 39, no. 7, pp. 1106-1111, 2000.

[6] H.-J. Bang, S.-M. Jun, and C.-G. Kim, "Stabilized interrogation and multiplexing techniques for fibre Bragg grating vibration sensors," Meas. Sci. Technol. vol. 16, pp. 813-820, 2005.

[7] W. Ecke, I. Latka, R.Willsch, A. Reutlinger, and R. Graue, "Fibre optic sensor network for spacecraft health monitoring," Meas. Sci. Technol., vol. 12, no. 7, pp. 974-980, 2001.

[8] L. Mescia and F. Prudenzano. "Advances on optical fiber sensors." Fibers, vol. 2, no. 1, pp 1-23, 2013.

[9] J. P. Yao, "Microwave photonics for high resolution and high speed interrogation of fiber Bragg grating sensors," Fiber Integrated Opt., vol. 34, no. 4, pp. 230-242, 2015.

[10] D. Chen, C. Shu, and S. He, "Multiple fiber Bragg grating interrogation based on a spectrum-limited Fourier domain mode-locking fiber laser," Opt. Lett., vol. 33, no. 13, pp. 1395-1397, 2008.

[11] E. J. Jung, C. Kim, M. Y. Jeong, M. K. Kim, M. Y. Jeon, W. Jung, and Z. Chen, "Characterization of FBG sensor interrogation based on a FDML 
wavelength swept laser," Opt. Express., vol. 16, no. 21, pp. 16552-16560, 2008.

[12] D. R. Solli, J. Chou, and B. Jalali, "Amplified wavelength-time transformation for real-time spectroscopy," Nature Photon., vol. 2, no. 1, pp. 48-51, 2008.

[13] K. Goda and B. Jalali, "Dispersive Fourier transformation for fast continuous single-shot measurements," Nature Photon., vol. 7, no. 2, pp. 102-112, 2013.

[14] P. V. Kelkar, F. Coppinger, A. S. Bhushan and B. Jalali, "Time-domain optical sensing," Electron. Lett. vol. 35, no. 19, pp. 1661-1662, 1999.

[15] J. Chou, Y. Han, and B. Jalali, "Time-wavelength spectroscopy for chemical sensing," IEEE Photon. Technol. Lett., vol. 16, no. 4, pp. 1140-1142, 2004.

[16] M. L. Dennis, M. A. Putnam, J. U. Kang, T.-E. Tsai, I. N. Duling, and E. J. Friebele, "Grating sensor array demodulation by use of a passively mode-locked fiber laser," Opt. Lett., vol. 22, no. 17, pp. 1362-1364, 1997.

[17] H. Y. Fu, H. L. Liu, X. Dong, H. Y. Tam, P. K. A. Wai and C. Lu, "High-speed fibre Bragg grating sensor interrogation using dispersion compensation fibre," Electron. Lett. vol. 44, no. 10, pp. 618-619, 2008.

[18] H. Xia, C. Wang, S. Blais, and J. P. Yao, "Ultrafast and precise interrogation of fiber Bragg grating sensor based on wavelength-to-time mapping incorporating higher-order dispersion," J. Lightw. Technol., vol. 28, no. 3, pp. 254-261, 2010.

[19] M. A. Muriel, J. Azana, and A. Carballar, "Real-time Fourier transformer based on fiber gratings," Opt. Lett., vol. 24, no. 1, pp. 1-3, 1999.

[20] C. Wang, "Dispersive Fourier transformation for versatile microwave photonics applications," Photonics, vol. 1, no. 4, pp. 586-612, 2014.

[21] G. Rodriguez, M. Jaime, F. Balakirev, C. H. Mielke, A. Azad, B. Marshall, B. M. La Lone, B. Henson, and L. Smilowitz, "Coherent pulse interrogation system for fiber Bragg grating sensing of strain and pressure in dynamic extremes of materials," Opt. Express, vol. 23, no. 11, pp. 14219-14233, 2015.

[22] W. Liu, M. Li, C. Wang, and J. P. Yao, "Real-time interrogation of a linearly chirped fiber Bragg grating sensor with improved resolution and signal-to-noise ratio," J. Lightwave Technol., vol. 29, no. 9, pp. 1239-1247, 2011.

[23] C. Wang and J. P. Yao, "Ultrafast and Ultrahigh-Resolution Interrogation of a Fiber Bragg Grating Sensor Based on Interferometric Temporal Spectroscopy," J. Lightwave Technol., vol. 29, no. 19, pp. 2927-2933, 2011.

[24] Y. Okabe, R. Tsuji, and N. Takeda, "Application of chirped fiber Bragg grating sensors for identification of crack locations in composites," Composites Part A: Applied Science and Manufacturing, vol. 35, no. 1, pp. 59-65, 2004.

[25] J. Gan, Y. Hao, Q. Ye, Z. Pan, H. Cai, R. Qu, and Z. Fang, "High spatial resolution distributed strain sensor based on linear chirped fiber Bragg grating and fiber loop ringdown spectroscopy," Opt. Lett., vol. 36, no. 6, pp. 879-881, 2011.

[26] M. Volanthen, H. Geiger, and J. P. Dakin, "Distributed grating sensors using low-coherence reflectometry," J. Lightwave Technol., vol. 15, no. 11, pp. 2076-2082, 1997.

[27] M. Hideak, H. Igawa, K. Kageyama, K. Ohta, I. Ohsawa, K. Uzawa, M. Kanai, T. Kasai, and I. Yamaguchi, "Distributed strain measurement with high spatial resolution using fiber Bragg gratings and optical frequency domain reflectometry," in 18th International Conference on Optical Fiber Sensors (OFS), 2006, p. ThE40.

[28] Y. Wang, J. Zhang, O. Coutinho, and J. Yao, "Interrogation of a linearly chirped fiber Bragg grating sensor with high resolution using a linearly chirped optical waveform," Opt. Lett., vol. 40, no. 21, pp. 4923-4926, 2015.

[29] C. Wang and J. P. Yao, "Photonic generation of chirped microwave pulses using superimposed chirped fiber Bragg gratings," IEEE Photon. Technol. Lett., vol. 20, no. 9-12, pp. 882-884, 2008.

[30] W. Liu, W. Li, and J. P. Yao, "Real-time interrogation of a linearly chirped fiber Bragg grating sensor for simultaneous measurement of strain and temperature," IEEE Photon. Technol. Lett., vol. 23, no. 18, pp. 1340-1342, 2011.

Eamonn J Ahmed received the B.Eng. degree in Electronics Engineering from GIK Institute, Topi, Pakistan in 2010, and MSc. Photonics from Friedrich
Schiller University, Jena, Germany in Feb 2013. He is currently working towards Ph.D. degree in Electrical Engineering at the University of Kent, Canterbury, UK.

Chao Wang (S’08-M'11) received the B.Eng. degree in opto-electrical engineering from Tianjin University, Tianjin, China, in 2002, the M.Sc. degree in optics from Nankai University, Tianjin, China, in 2005, and the Ph.D. degree in electrical and computer engineering from the University of Ottawa, Ottawa, ON, Canada, in 2011.

From 2011 to 2012, he was a Postdoctoral Fellow with the Photonics Laboratory, University of California at Los Angeles, Los Angeles, CA, USA. He is currently a Lecturer (Assistant Professor) with the School of Engineering and Digital Arts, University of Kent, Canterbury, U.K. His research interests include microwave photonics, ultrafast imaging, optical communications, optical sensing, and biophotonics for scientific and engineering applications.

Dr. Wang is a member of IEEE Photonics Society, the IEEE Microwave Theory and Techniques Society (IEEE MTT-S) and the Optical Society of America (OSA). He was the recipient of the SPIE Scholarship in Optical Science and Engineering (2008), the IEEE Photonics Society (formerly LEOS) Graduate Student Fellowship (2009), the IEEE MTT-S Graduate Fellowship (2010), the Vanier Canada Graduate Scholarship (2009), the Chinese Government Award for Outstanding Self-Financed Students Abroad (2009), the Natural Sciences and Engineering Research Council (NSERC) of Canada Postdoctoral Fellowship (2010), and the EU Marie-Curie Career Integration Grant (2014).

Dejun Feng received the B.S. degree in physics from Qufu Normal University, China, in 1995, the M.S. degree in optical holography information processing from Beijing Normal University, China, in 1998, and the Ph.D. degree in Optics from the Institute of Modern Optics, Nankai University, China, in 2001. He was a post-doctoral fellow with KyungHee University, Korea, from 2001 to 2003. After this he moved to the University of Tokyo as a post-doctoral fellow for two years. He is currently a full Professor at Shandong University, China. From June 2015 to June 2016, he is a visiting Professor in the University of Kent, UK. His research interests are mainly on fiber grating fabrication, optical fiber sensors and fiber lasers.

Zhijun Yan received the B.S. and M.S. degrees in condensed state physics from Lanzhou University, China, in 2003 and 2006, respectively, and the Ph.D. degree in electronics and electrical engineering from Aston University, U.K., in 2013. From 2013 to 2016, he was a Research Fellow with the Aston Institute of Photonic Technologies. He has authored over 50 articles and three inventions. Since 2016, he has been with the State Key Laboratory of Transient Optics and Photonics, Xi'an Institute of Optics and Precision Mechanics, Chinese Academy of Sciences, China.

His research interests include optical fiber device design, optical fiber sensing, and optical fiber laser systems.

Lin Zhang received the Ph.D. degree from Sussex University, U.K., in 1990. She was a Post-Doctoral Research Fellow with Sussex University, where she was involved in passive and active planar optical waveguide devices. In 1994, she joined the Aston Institute of Photonic Technologies (formerly the Photonics Research Group), School of Engineering and Applied Science, Aston University, U.K., where she was appointed as a Lecturer in 2001 and the Chair Professor in 2005. Her research interests are primarily in the field of advanced photonic devices, techniques, and applications. She has contributed mainly in the areas of optical fiber grating technologies and applications in optical communications, signal processing, microwave photonics, fiber lasers, and smart sensing. Her research output so far includes 450 papers published in peer-review journals and international conferences. She has co-authored three special technical books. 